\title{
Misdiagnosed recurrent multiple Kimura's disease: A case report and review of the literature
}

\author{
XUESHENG LI ${ }^{1}, \mathrm{JING}_{\mathrm{WANG}}^{2}, \mathrm{HONGBO} \mathrm{LI}^{2}$ and MING ZHANG ${ }^{2}$ \\ ${ }^{1}$ Department of Prosthodontics, Hainan Stomatological Hospital, Haikou, Hainan 570105; ${ }^{2}$ Department of Stomatology, \\ Hainan Branch of Chinese People's Liberation Army General Hospital, Sanya, Hainan 572013, P.R. China
}

Received September 5, 2018; Accepted December 7, 2018

DOI: $10.3892 /$ mco.2018.1793

\begin{abstract}
Kimura's disease (KD) is a rare condition, with only a few cases reported to date, mainly in Asian patients. We herein present the case of a 48-year-old man with KD who presented with recurrent masses in the right parotid gland and neck region over a 15 -year period. The masses were not accompanied by pain, or significant functional or neurosensory dysfunction. The results of the laboratory tests revealed an increased eosinophil count and markedly elevated serum IgE levels. On magnetic resonance imaging examination, a widespread abnormal signal was detected in the area of the lesions; the contrast-enhanced scan revealed inhomogeneous enhancement, with partial involvement of the sternocleidomastoid muscle and the parotid gland. The patient underwent surgical resection of the right parotid and neck masses, and the postoperative pathological examination revealed eosinophilic hyperplastic lymphogranuloma, also referred to as KD. This presented case and review of the relevant literature aim to improve our understanding of KD in order to increase the accuracy of diagnosis, reduce the misdiagnosis rate and ensure proper treatment of this rare disease.
\end{abstract}

\section{Introduction}

Kimura's disease (KD) was first reported in China by Kim in 1937 as eosinophilic hyperplastic lymphogranuloma (1). Until 1948, the disease was widely recognized in Japan and systematically described by Professor Kimura as 'unusual granulations combined with hyperplastic changes in lymphoid tissue' $(2,3)$. KD is a chronic disease and its etiology has not been fully elucidated to date. Patients suffering from this condition mainly present with solitary or multiple painless masses in the maxillofacial and other regions, which often

Correspondence to: Dr Ming Zhang, Department of Stomatology, Hainan Branch of Chinese People's Liberation Army General Hospital, 80 Linwang Road, Sanya, Hainan 572013, P.R. China E-mail: doctorzm@163.com

Key words: Kimura's disease, eosinophilic hyperplastic lymphogranuloma, eosinophil granulocyte recur after treatment $(4,5)$. Clinically, KD is always accompanied by enlarged regional lymph nodes, eosinophilia and markedly elevated serum IgE levels $(2,5)$. This disease has been most commonly diagnosed in middle-aged individuals in the Far East, for example China and Japan (6-8). Thus far, only 200 cases have been reported worldwide. We herein report a rare case of KD in a 48-year-old man and review the relevant literature to help improve the level of clinicians' knowledge regarding the diagnosis and treatment of this disease.

\section{Case report}

A 48-year-old man was admitted to the Hainan Branch of Chinese People's Liberation Army General Hospital (Sanya, China) in December 2017, with recurrent masses in the right parotid gland and neck region for $\sim 15$ years. The patient was in good overall condition and had no other complaints, such as pain, swelling, local numbness or xerostomia. There were no reported allergies or other systemic manifestations. The patient had undergone surgical resection of the masses twice (1997 and 2000), but the masses recurred both times after $\sim 6$ months. Both postoperative pathological diagnoses were lymphadenitis.

Physical examination revealed maxillofacial asymmetry. Hard, mobile masses were identified in the region of the right parotid gland and neck, arranged longitudinally. On palpation, the borders of the masses were clear, without adhesions to the surrounding tissue; the total size was $15 \times 10 \times 3 \mathrm{~cm}$. Masses were also identified in the left supraclavicular fossa, the right forearm near the wrist joint and the left medial upper arm, sized $5 \times 5 \times 2,4 \times 3 \times 2$ and $4 \times 3 \times 1 \mathrm{~cm}$, respectively (Fig. 1). In addition, multiple enlarged lymph nodes were palpated in the submandibular area and the neck region.

Reviewing the results of the laboratory tests, with an increased eosinophil percentage (0.55\%; normal, 0.01-0.05\%), markedly elevated serum IgE levels $(27,100 \mathrm{IU} / \mathrm{ml}$; normal, $0-100 \mathrm{IU} / \mathrm{ml})$ and leukocyte count $\left(21.78 \times 10^{9} / 1\right.$; normal, $\left.3.5 \times 10^{9} / 1\right)$, and decreased neutrophil percentage $(0.28 \%$; normal, $0.50-0.70 \%$ ). The results of other investigations, including routine urinalysis, liver and kidney function tests, were within normal limits. Chest radiography was also normal.

Ultrasound examination of the right parotid gland region revealed uneven echogenicity, consistent with a high likelihood of a benign tumor (Fig. 2); a low-echogenicity 

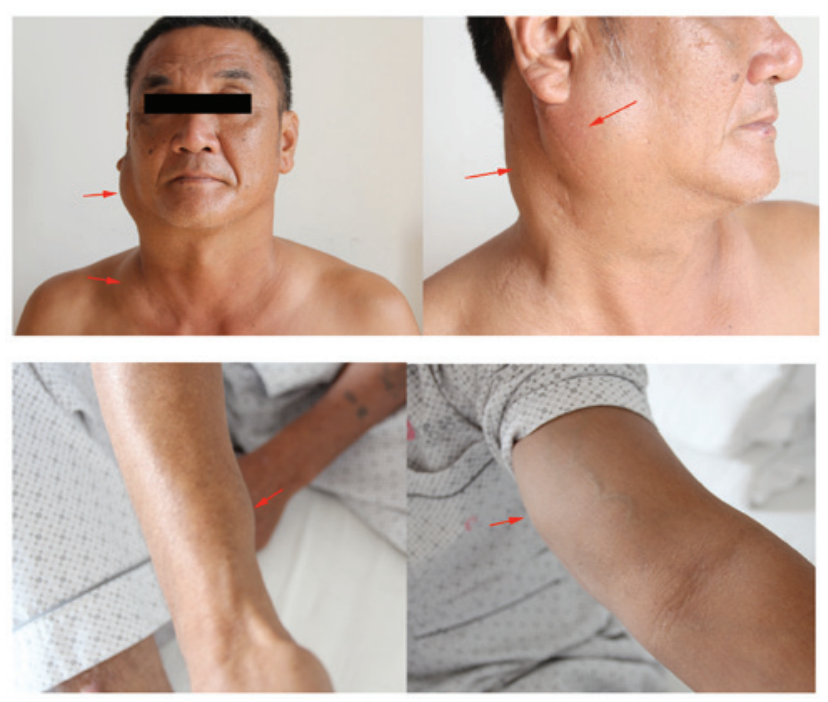

Figure 1. The patient who presented with painless masses (arrows) in the region of the right parotid gland and neck, the left supraclavicular fossa, the right forearm near the wrist joint and the left medial upper arm.

nodule was identified in the right submaxillary region, which was considered to be a reactive hyperplastic lymph node. On magnetic resonance imaging (MRI) examination, a widespread abnormal signal was detected in the right parotid gland and submaxillary region, and a contrast-enhanced scan revealed inhomogeneous enhancement; the right side of the sternocleidomastoid muscle and the parotid gland were partially involved, with half of the lesions wrapped around the carotid sheath (Fig. 3); multiple enlarged lymph nodes were also identified displaying obvious enhancement.

A detailed surgical plan was formulated and, following a full preoperative preparation, the right parotid gland and neck masses were resected under general anesthesia, together with the right superficial parotid lobe (Fig. 4).

Postoperative pathological examination established the diagnosis of eosinophilic hyperplastic lymphogranuloma, also referred to as KD (Fig. 5), with lesions involving the parotid gland and lymph node tissue.

The patient was administered $25 \mathrm{mg}$ prednisone twice daily for 2 weeks after the definitive diagnosis. On repeat blood tests, the eosinophil count returned to normal levels, and the masses of the left supraclavicular fossa, right forearm and left medial upper arm notably decreased in size. Therefore, the initial treatment was successful in controlling the disease. Next, the patient will be advised to undergo radiotherapy (total dose, 20-50 Gy; 1.8-2.0 Gy per day, five days a week, over the course of 2-5 weeks).

\section{Discussion}

$\mathrm{KD}$ is a rare condition, with $<300$ reported cases to date. Based on these cases, KD is rarely observed in Europe and America $(9,10)$, but frequently occurs in East Asian and Southeast Asian populations (11-14), with China and Japan being the most common. The pathogeny of KD remains unknown. The minimum age reported is 5 years, but it mostly occurs in middle-aged men (15-17). Women only account for a small proportion of the patients, with a male:female ratio of $3: 1$ (18). Clinically, KD has a specific predilection for the

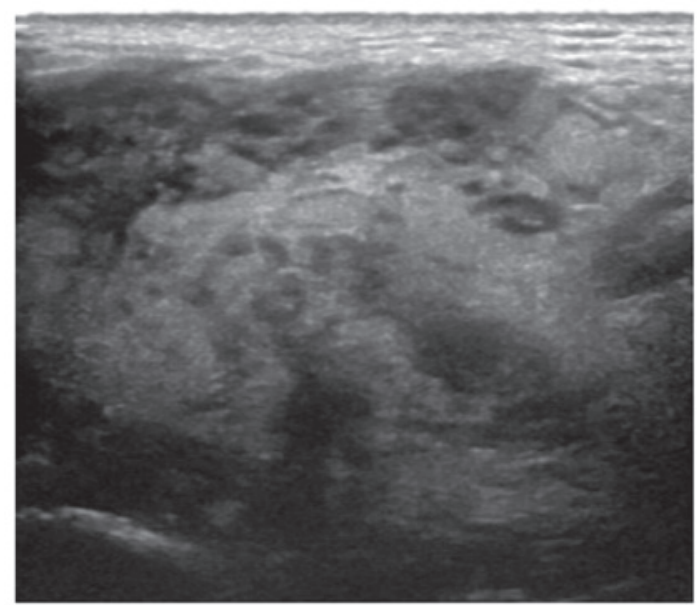

Figure 2. Ultrasound of the right parotid gland region showing uneven echogenicity.

head, neck $(5,10)$ and limbs, with the lesions most commonly developing in the soft tissue, whereas maxillofacial salivary glands and lymph nodes may also be affected. Patients with KD disease often have a long course, manifesting solitary or multiple soft tissue masses. KD is usually associated with peripheral blood eosinophilia and markedly elevated IgE levels.

The etiology of KD remains unknown $(4,5,10,13)$. It has been confirmed that there is no correlation with tuberculosis, bacterial, fungal or viral infections, poisoning or syphilis (19). Most scholars tend to consider KD as an IgE-mediated type I allergy and inflammatory disease (20), and this hypothesis is supported by the increased eosinophil count and IgE levels in the peripheral blood $(21,22)$. Googe et al reported increased IgE levels in KD patients with renal involvement, which supports the theory that KD is an immune response (23); moreover, clinical application of steroid therapy appears to be effective, further supporting this theory. In recent years, Ohta et al measured Th1/Th2 and Tc1/Tc2 lymphocyte subsets by flow cytometry in KD patients, and interestingly observed 


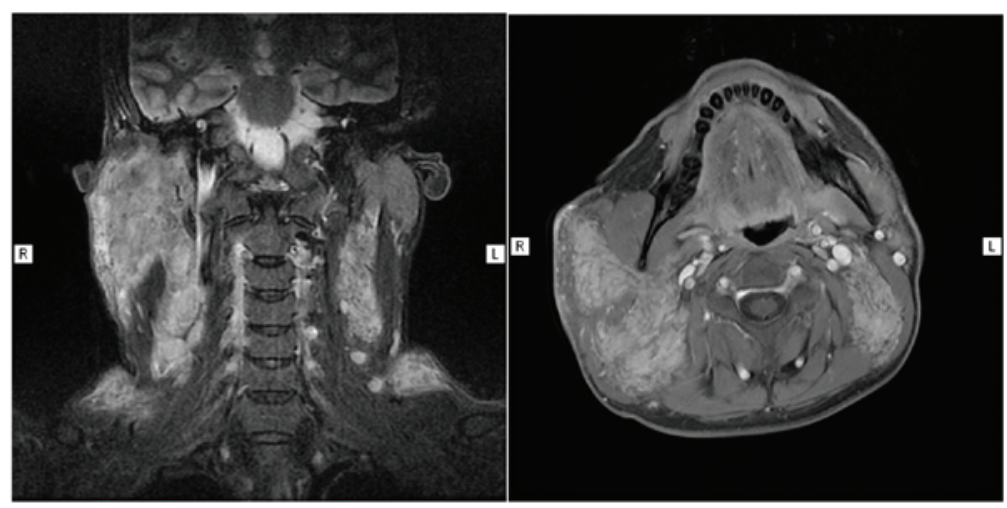

Figure 3. Magnetic resonance imaging examination showing widespread abnormal signal was detected. Administration of contrast material revealed uneven enhancement.
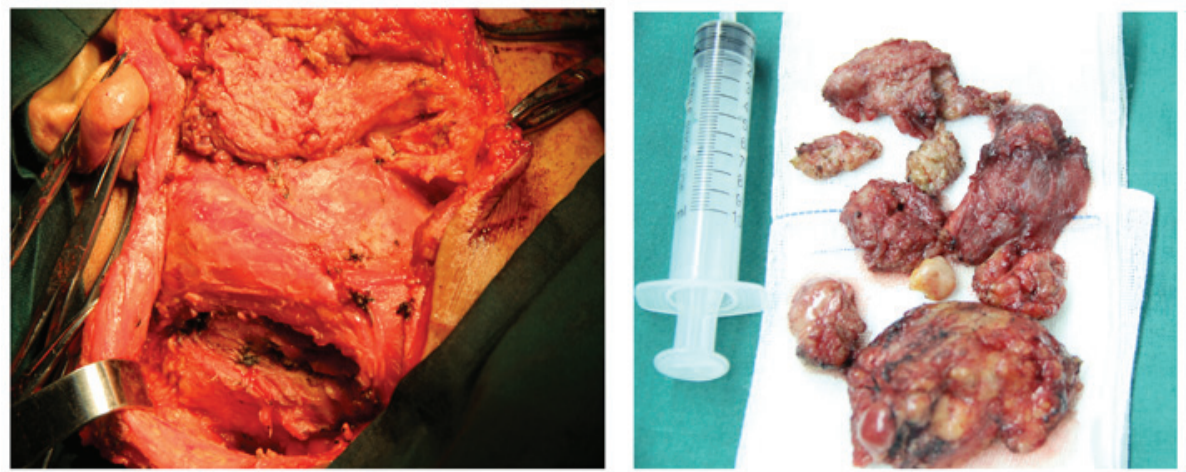

Figure 4. Intraoperative view (left panel) and resected specimens (right panel).

that Th2 and Tc1 cell numbers increased significantly in patients with KD compared with the healthy control group; therefore, they reported that Th2 and Tc1 cells appear to play an important role in the pathogenesis of KD (24). Other studies demonstrated that the release of cytokines in patients with KD can increase the permeability of the glomerular basement membrane, causing proteinuria, which may ultimately cause renal damage $(25,26)$. Chim et al considered a clonal T-cell lymphoproliferative disorder as the possible cause of $\mathrm{KD}$; however, this result was from a single case study, and the etiological analysis remains speculative (27).

KD patients are typically men in their 30s and the highest incidence of this disease worldwide is observed in the Asian population. Although Asian and non-Asian patients have certain characteristics in common, it has been reported that non-Asian patients do not generally exhibit involvement of the salivary glands (10); by contrast, in Asian patients KD has a predilection for pre-auricular, parotid and submandibular salivary glands, the neck and the maxillofacial region. The majority of the patients present with firm, painless, single or multiple subcutaneous masses, progressively increasing in size. Mass size may vary greatly, ranging from 1 to $7 \mathrm{~cm} \mathrm{(28).}$ In addition, multiple regional lymph nodes are involved, manifesting as a single or multiple fused nodules. Although KD mostly involves the maxillofacial region, involvement of other sites has also been reported, including the axilla, trunk, kidney, arm, nerves, orbit, spermatic cord and groin $(6,29)$. In the case presented herein, the patient had a right parotid swelling,

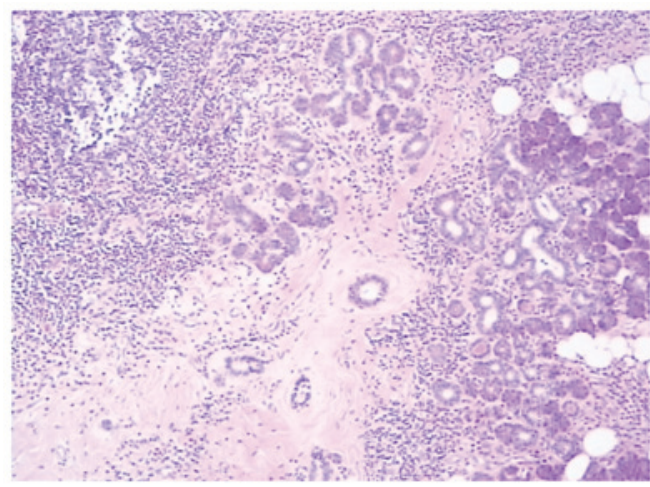

Figure 5. Diffuse infiltration of the parotid gland by eosinophil granulocytes and lymphocytes (hematoxylin and eosin staining; magnification, $\mathrm{x} 40$ ).

along with multiple supraclavicular fossa and arm masses for 15 years. The skin overlying the KD lesions was not inflamed, although mild itching was occasionally reported. With disease progression, skin pigmentation, thickening, local erosion and ulceration may develop, sometimes affecting the whole body in severe cases. Some scholars report that renal involvement is a common systemic manifestation that may affect up to $60 \%$ of the patients $(5,25,30,31)$. In addition, the disease may be complicated by asthma, fatigue, fever, allergic rhinitis, atopic dermatitis, urticaria and other allergic symptoms (20). KD is generally benign and self-limited, with a prolonged course. On long-term follow-up, malignant transformation has not been 
reported. The overall prognosis is good, but recurrence is common.

KD patients almost always have marked eosinophilia and elevated serum IgE levels. Some studies report that blood eosinophil counts are closely associated with the size of the lesion, i.e., the bigger the lesion, the higher the eosinophil count $(32,33)$. Physical examination along with US, CT and MRI may help determine the characteristics, boundaries and blood supply of the mass, as well as the presence of lymph node involvement. On US, the mass may exhibit heterogeneous or homogeneous echogenicity (34), occasionally displaying increased vascularity. On CT scans, the lesions are strongly enhanced, reflecting their vascular nature; lymphadenopathy is reported among the typical findings. On MRI, the lesion exhibits intermediate to high signal intensity on T1-weighted images and hyperintense signals on T2-weighted images $(35,36)$. Therefore, imaging may be a useful way of demonstrating the morphology and extent of the lesion, as well as its association with adjacent structures (8).

There are currently no uniform diagnostic criteria for KD. The following characteristics should raise suspicion of KD: i) Young male, with head and/or neck painless mass; ii) local enlarged lymph nodes; iii) long history; iv) parts of body other than the head and neck displaying multiple painless masses, accompanied by pruritus of the overlying skin; v) increased blood eosinophil count and serum IgE level (a high reference value is needed to make a correct diagnosis); and vi) CT and MRI showing a wide range of lesions and multiple enlarged lymph nodes. Based on these clinical manifestations and blood test results, the diagnosis of KD may be preliminarily considered, but the final diagnosis relies on pathological examination. The histopathological characteristics of KD include follicular hyperplasia, eosinophilic infiltrates and proliferation of postcapillary venules $(2,10,12,23)$.

Clinically, the differential diagnosis of KD may include angiolymphoid hyperplasia with eosinophilia (ALHE), Hodgkin's lymphoma, angioimmunoblastic T-cell lymphoma, Langerhans cell histiocytosis, florid follicular hyperplasia, Castleman's disease, dermatopathic lymphadenopathy, sinonasal eosinophilic angiocentric fibrosis, drug reactions and parasitic infections. In the literature, the most common misdiagnosis is ALHE (10,37); however, patients with ALHE have normal $\operatorname{IgE}$ levels and no kidney damage $(3,9,38,39)$.

In conclusion, the prognosis for KD is quite favorable, but may recur frequently in the original location (4), with a recurrence rate of up to $25 \%$ (40), posing a major challenge to the physician and patient. A standard treatment for KD has not yet been established. The goal of treatment currently is to maintain appearance and functionality, while preventing recurrence and long-term sequelae, including nephritis and myocarditis.

Although surgery is the most widely used treatment method and it can help reach a definite diagnosis $(32,33,35,41,42)$, other options include radiotherapy, systemic corticosteroids, cytotoxic agents, cyclosporin and pentoxifylline $(32,33,42)$. Oral corticosteroids are usually recommended in cases of symptomatic nephrotic syndrome (26) and, in order to prevent relapse and reduce the long-term side effects of steroid therapy, postoperative radiation therapy may be used (low-dose local irradiation, 25-30 Gy) $(43,44)$. Reportedly, the recurrence rate appears to be lower if two treatment modalities are combined (41). Recently, anti-IgE therapy has been introduced (45), and the size of lesion and peripheral blood eosinophil count of KD patients were reported to decrease following anti-IgE therapy.

In conclusion, KD is a chronic disorder of unknown etiology. In clinical practice, KD is easily misdiagnosed as other inflammatory lesions or benign tumors of the head and neck, so more thorough diagnostic workup is required. Only through a careful medical history and comprehensive clinical examination, combined with laboratory, imaging and histopathological examinations, can a definitive diagnosis be reached and individualized treatment administered.

\section{Acknowledgements}

Not applicable.

\section{Funding}

The present study was supported by the Hainan Key Research and Development Program (grant no. ZDYF2016170) and the Clinical Research Support Fund of PLA General Hospital (grant no. 2017FC-WJFWZX-22).

\section{Ethics approval and consent to participate}

Not applicable.

\section{Patient consent for publication}

The patient provided consent for publication of the case details and associated images.

\section{Availability of data and materials}

Not applicable.

\section{Authors' contributions}

XL took care of the patient, wrote the medical records and wrote the manuscript. JW and HL collected relevant medical records and assisted with writing. MZ was responsible for surgical treatment and paper revision. All the authors have read and approved the final version of this manuscript.

\section{Competing interests}

The authors declare that they have no competing interests to disclose.

\section{References}

1. Kim HT: Eosinophilic hyperplastic lymphogranuloma, comparison with Mikulicz's disease. Chin Med J 23: 699, 1937.

2. Kung IT, Gibson JB and Bannatyne PM: Kimura's disease: A clinico-pathological study of 21 cases and its distinction from angiolymphoid hyperplasia with eosinophilia. Pathology 16: 39-44, 1984.

3. Kimura T and Yoshimura S: Unusual granuloma combined with hyperplastic changes in lymphatic tissues. Trans Soc Path Jpn 13: 179-180, 1948.

4. Abuel-Haija M and Hurford MT: Kimura disease. Arch Pathol Lab Med 131: 650-651, 2007.

5. Kuo TT, Shih LY and Chan HL: Kimura's disease. Involvement of regional lymph nodes and distinction from angiolymphoid hyperplasia with eosinophilia. Am J Surg Pathol 12: 843-854, 1988. 
6. Takeishi M, Makino Y, Nishioka H, Miyawaki T and Kurihara K Kimura disease: Diagnostic imaging findings and surgical treatment. J Craniofac Surg 18: 1062-1067, 2007.

7. Iwai H, Nakae K, Ikeda K, Ogura M, Miyamoto M, Omae M, Kaneko T and Yamashita T: Kimura disease: Diagnosis and prognostic factors. Otolaryngol Head Neck Surg 137: 306-311, 2007.

8. Park SW, Kim HJ, Sung KJ, Lee JH and Park IS: Kimura disease: $\mathrm{CT}$ and MR imaging findings. AJNR Am J Neuroradiol 33: 784-788, 2012.

9. Iguchi Y, Inoue T, Shimono M, Yamamura T, Shigematsu T and Takahashi S: Kimura's disease and its relation to angiolymphoid hyperplasia with eosinophilia: Report of three cases and review of the literature. J Oral Pathol 15: 132-137, 1986.

10. Chen H, Thompson LD, Aguilera NS and Abbondanzo SL: Kimura disease: A clinicopathologic study of 21 cases. Am J Surg Pathol 28: 505-513, 2004

11. Gumbs MA, Pai NB, Saraiya RJ, Rubinstein J, Vythilingam L and Choi YJ: Kimura's disease: A case report and literature review. J Surg 70: 190-193, 1999.

12. Hui PK, Chan JK, Ng CS, Kung IT and Gwi E: Lymphadenopathy of Kimura's disease. Am J Surg Pathol 13: 177-186, 1989.

13. Urabe A, Tsuneyoshi M and Enjoji M: Epithelioid hemangioma versus Kimura's disease. A comparative clinicopathologic study. Am J Surg Pathol 11: 758-766, 1987.

14. Xu X, Fu J, Fang Y and Liang L: Kimura disease in children: A case report and a summary of the literature in Chinese. J Pediatr Hematol Oncol 33: 306-311, 2011.

15. Francis IC, Kappagoda MB, Smith J and Kneale K: Kimura's disease of the orbit. Ophthal Plast Reconstr Surg 4: 235-239, 1988

16. Seregard S: Angiolymphoid hyperplasia with eosinophilia should not be confused with Kimura's disease. Acta Ophthalmol Scand 79: 91-93, 2001.

17. Takagi K, Harada T and Ishikawa E: Kimura's disease (eosinophilic lymphfolliculoid granuloma). Nihon Rinsho 51: 785-788, 1993 (In Japanese).

18. Tseng CF, Lin HC, Huang SC and Su CY: Kimura's disease presenting as bilateral parotid masses. Eur Arch Otorhinolaryngol 262: 8-10, 2005

19. Asadi AK: Angiolymphoid hyperplasia with eosinophilia Dermatol Online J 8: 10, 2002

20. Kimura Y, Pawankar R, Aoki M, Niimi Y and Kawana S: Mast cells and T cells in Kimura's disease express increased levels of interleukin-4, interleukin-5, eotaxin and RANTES. Clin Exp Allergy 32: 1787-1793, 2002.

21. Oguz KK, Ozturk A and Cila A: Magnetic resonance imaging findings in Kimura's disease. Neuroradiology 46: 855-858, 2004.

22. Choi JA, Lee GK, Kong KY, Hong SH, Suh JS, Ahn JM, Lee YJ, Cho KH, Park JG, Choi JY, et al: Imaging findings of Kimura's disease in the soft tissue of the upper extremity. AJR Am J Roentgenol 184: 193-199, 2005.

23. Googe PB, Harris NL and Mihm MC Jr: Kimura's disease and angiolymphoid hyperplasia with eosinophilia: Two distinct histopathological entities. J Cutan Pathol 14: 263-271, 1987.

24. Ohta N, Fukase S, Suzuki Y, Ito T, Yoshitake H and Aoyagi M: Increase of Th2 and Tc1 cells in patients with Kimura's disease. Auris Nasus Larynx 38: 77-82, 2011.

25. Rajpoot DK, Pahl M and Clark J: Nephrotic syndrome associated with Kimura disease. Pediatr Nephrol 14: 486-488, 2000.

26. Uysal IO, Eryilmaz MA, Salk I and Abasiyanik F: Kimura disease in the parotid gland. J Craniofac Surg 22: 337-338, 2011.

27. Chim CS, Fung A, Shek TW, Liang R, Ho WK and Kwong YL: Analysis of clonality in Kimura's disease. Am J Surg Pathol 26 1083-1086, 2002.
28. Dik VK, van de Wiel BA and Vasmel WL: Kimura's disease of the parotid glands and multiple cervical lymph nodes. Neth J Med 68: 175-177, 2010.

29. Gopinathan A and Tan TY: Kimura's disease: Imaging patterns on computed tomography. Clin Radiol 64: 994-999, 2009.

30. Chan TM, Chan PC, Chan KW and Cheng IK: IgM nephropathy in a patient with Kimura's disease. Nephron 58: 489-490, 1991.

31. Akosa AB, Sherif A and Maidment CG: Kimura's disease and membranous nephropathy. Nephron 58: 472-474, 1991.

32. Sun QF, Xu DZ, Pan SH, Ding JG, Xue ZQ, Miao CS, Cao GJ and Jin DJ: Kimura disease: Review of the literature. Intern Med J 38: 668-672, 2008

33. Sakamoto M, Komura A and Nishimura S: Hematoserological analysis of Kimura's disease for optimal treatment. Otolaryngol Head Neck Surg 132: 159-160, 2005.

34. Ragu R, Eng JY and Azlina AR: Kimura's disease of the parotid: A complete clinical-radiological-pathology report. Med J Malaysia 69: 199-201, 2014

35. Som PM and Biller HF: Kimura disease involving parotid gland and cervical nodes: CT and MR findings. J Comput Assist Tomogr 16: 320-322, 1992.

36. Takahashi S, Ueda J, Furukawa T, Tsuda M, Nishimura M, Orita H, Tsujimura T and Araki Y: Kimura disease: CT and MR findings. AJNR Am J Neuroradiol 17: 382-385, 1996.

37. Buggage RR, Spraul CW, Wojno TH and Grossniklaus HE: Kimura disease of the orbit and ocular adnexa. Surv Ophthalmol 44: 79-91, 1999.

38. Katagiri K, Itami S, Hatano Y, Yamaguchi T and Takayasu S: In vivo expression of IL-4, IL-5, IL-13 and IFN-gamma mRNAs in peripheral blood mononuclear cells and effect of cyclosporin A in a patient with Kimura's disease. Br J Dermatol 137: 972-977, 1997.

39. Kimura T and Yoshimura S: E. I: Unusual granuloma combined with hyperplastic changes in lymphatic tissues. Trans Soc Path Jpn 37: 179, 1948.

40. Allen PW, Ramakrishna B and MacCormac LB: The histiocytoid hemangiomas and other controversies. Pathol Annu 27: 51-87, 1992.

41. Hiwatashi A, Hasuo K, Shiina T, Ohga S, Hishiki Y, Fujii K and Ishitoya J: Kimura's disease with bilateral auricular masses. AJNR Am J Neuroradiol 20: 1976-1978, 1999.

42. Shetty AK, Beaty MW, McGuirt WF Jr, Woods CR and Givner LB: Kimura's disease: A diagnostic challenge. Pediatrics 110: e39, 2002

43. Itami J, Arimizu N, Miyoshi T, Ogata $\mathrm{H}$ and Miura K: Radiation therapy in Kimura's disease. Acta Oncol 28: 511-514, 1989.

44. Kim GE, Kim WC, Yang WI, Kim SK, Oh WY, Suh HS, Hahn JS and Park CS: Radiation treatment in patients with recurrent Kimura's disease. Int J Radiat Oncol Biol Phys 38: 607-612, 1997.

45. Nonaka M, Sakitani E and Yoshihara T: Anti-IgE therapy to Kimura's disease: A pilot study. Auris Nasus Larynx 41: 384-388, 2014.

(i) $\Theta$ This work is licensed under a Creative Commons Attribution-NonCommercial-NoDerivatives 4.0 International (CC BY-NC-ND 4.0) License. 